\title{
Energy dispersive micro-XRF Bragg-pattern visualization - Laue Mapping
}

\author{
Max Buegler ${ }^{1}$, Roald Tagle ${ }^{1}$, Falk Reinhardt ${ }^{1}$, Andrew Menzies ${ }^{1}$ and Tina Hill ${ }^{2}$ \\ ${ }^{1}$ Bruker Nano GmbH, Berlin, Berlin, Germany, ${ }^{2}$ Bruker Nano GmbH, United States
}

In this contribution we discuss the fast acquisition of X-ray maps and the subsequent visualization of Bragg-peaks. The data is acquired utilizing an M4 TORNADO Plus equipped with two sources-Rh with polycapillary optic and $\mathrm{W}$ with collimator changer. The hard- and software of the M4 allows for measuring with either source, or one or both detectors at the same time. It is also possible to map a sample utilizing both detectors and retroactively separate the data for the two detectors. This development enables a differential analytical approach to quantification and visualization.

Fluorescence provides insight into the composition of a sample and current advanced energy dispersive detectors and high brilliance excitation allow for fast scanning and mapping. For the last century, crystalline artefacts in acquired XRF spectra such as Bragg peaks were considered a significant hindrance to quantitative analysis. Solutions with some success include suppression of artefacts with primary filters or use of monochromatic excitation. New solutions such as collecting a full spectrum for each pixel, improved fitting of fluorescence peaks (regardless of other (broader) artefacts beneath), as well as the analytical tools to easily visualize the intensity of such artefacts across an entire map translates into utilization of micro-XRF for quickly assessing composition and size and distribution of crystallites... all from the same dataset. The M4 TORNADO facilitates this faster and more precise investigation by acquiring data from two opposing detectors synchronously while allowing for extraction of information from each detector separately. Utilizing this separate data from both detectors, a map with drastically reduced Bragg peak intensity can be extracted for quantitative analysis by analyzing each individual pixel and channel at their lowest value ('minimum pixel spectrum'). At the same time, it is possible to analyze crystal structure from the same database, employing the sum or either of the two detectors independently.

Examples of Laue maps from applications in a variety of fields are presented. One essential field which values this type of information is metallurgy. In rolled metals the crystallites often grow significantly in size during the rolling process. At the intersection of those crystallites the structure of the material is distorted. Often there will be additional dangling bonds and 'cracks' in the lattice which show lower resistance against corrosion and often show reduced mechanical stability at the defects. Reduced mechanical stability is of concern in sheet metals and in many welded materials. Visualization of crystallite size and composition together may lead to better failure analysis of parts. A large welding joint between two large pieces of steels will be presented and the results discussed.

Here we show that micro-XRF mapping allows for a quick assessment of crystalline quality over large areas of sample. Micro-XRF permits scanning large areas in mere hours, where one can easily spend days collecting data for a proper reciprocal space map. This was demonstrated by C. Guguschev et al. on a large single crystal of SrTiO3 at the Leibniz Institute for Crystal Growth. In this study, an area of 700 $\mathrm{mm}^{2}$ was investigated with 1.1 million points in about 16 hours. 1

Another field of application in which this information is important is solar cell technology. Here the size of crystallites and the density of grain boundaries have a significant effect on the internal resistance and the resulting wall plug efficiency. In some cases, the top layer of crystallites can be investigated optically, but to understand the bulk of the material a deeper insight is necessary. In micro-XRF information depth strongly depends on the photon energy of the investigated element as well as the density of the matrix. 
For Silicon, an important element in solar cell technology, this results in an information depth for Braggpeaks of about $6 \mu \mathrm{m}$ at $4 \mathrm{keV}$ and up to $185 \mu \mathrm{m}$ at $14 \mathrm{keV}$.

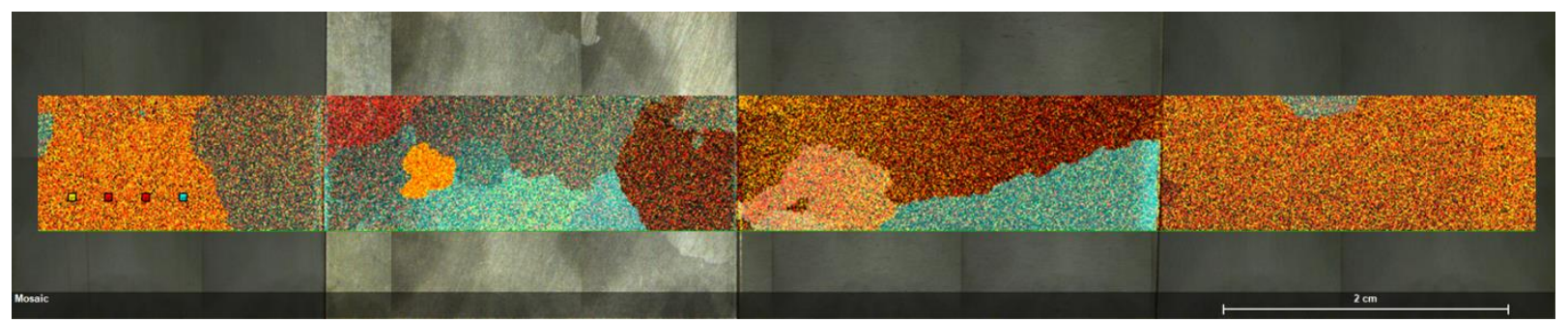

Figure 1. Lauemap of 4 pieces of sheet metal at different stages of coating. Note that even under a glassy coating on steel, it is possible to resolve the Bragg peaks from the underlying sheet metal. Left is the complete stack of coatings, second is the uncoated, third and forth sucesssive steps of the coating.
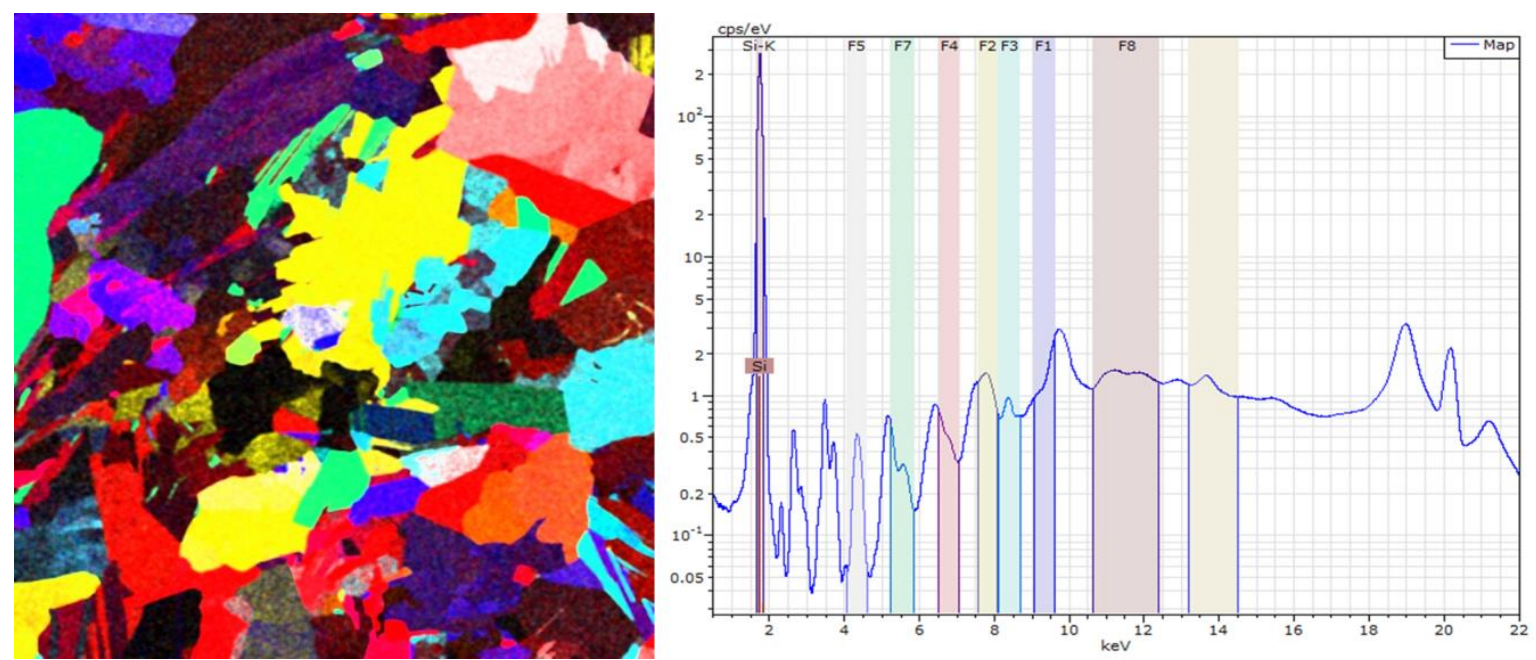

Figure 2. A Laue map of a polycrystalline sheet of silicon, visualizing the appearance and disappearance of Bragg peaks across the sample surface. Optical imaging allows for surfaccial inspection however, micro-XRF allows investigation deeper into the volume of the sample.

\section{References}

${ }^{1}$ C. Guguschev, R. Tagle, U. Juda, A. Kwasniewski 'Microstructural investigations of SrTiO3 single crystals and polysilicon using a powerful new X-ray diffraction surface mapping technique'; J. Appl. Cryst. (2015). 48, 1883-1888; https://doi.org/10.1107/S1600576715019949 